\title{
Scattering of quantum wave packets by shallow potential islands: A quantum lens
}

\author{
Arseni Goussev ${ }^{1,2}$ and Klaus Richter ${ }^{3}$ \\ ${ }^{1}$ Department of Mathematics and Information Sciences, Northumbria University, Newcastle Upon Tyne NE1 8ST, United Kingdom \\ ${ }^{2}$ Max Planck Institute for the Physics of Complex Systems, Nöthnitzer Straße 38, D-01187 Dresden, Germany \\ ${ }^{3}$ Institute for Theoretical Physics, University of Regensburg, D-93040 Regensburg, Germany
}

(Received 21 March 2013; published 28 May 2013)

\begin{abstract}
We consider the problem of quantum scattering of a localized wave packet by a weak Gaussian potential in two spatial dimensions. We show that, under certain conditions, this problem bears close analogy with that of focusing (or defocusing) of light rays by a thin optical lens: Quantum interference between straight paths yields the same lens equation as for refracted rays in classical optics.
\end{abstract}

DOI: 10.1103/PhysRevE.87.052918

PACS number(s): 05.45.Mt, 03.65.Nk, 03.65.Sq, 42.25.Fx

\section{INTRODUCTION}

The intrinsic connection between the motion of classical particles on the one hand and the propagation of quantum matter waves on the other has occupied the minds of scientists since the early days of quantum theory [1]. Invaluable contributions of Ehrenfest, Van Vleck, Feynman, Gutzwiller, and Maslov, among many others, have shaped our current understanding of quantum wave propagation in terms of interference of classical trajectories. However, a number of important questions concerning quantum-classical correspondence remain open. These questions fall under the scope of the area of mathematical physics known as quantum chaos [2-4].

The motion of a classical particle can be conveniently described by means of a phase-space trajectory. The Heisenberg uncertainty principle, however, does not allow for the notion of the classical trajectory to be directly carried over to quantum theory. One natural extension of the classical concept of a point in the phase space is provided by a localized quantum wave packet that can be parametrized by its mean position and momentum, and dispersion quantifying the phase-space extent of the wave packet. According to the Ehrenfest theorem [5], the time evolution of the mean position and momentum is governed, for short enough times, by the classical equations of motion. In other words, the wave-packet center follows the corresponding classical trajectory.

An issue of the wave-packet spreading, i.e., how the dispersion depends on time, is, however, much more complex. Loosely speaking, there are two main mechanisms of the spreading: (i) a classical-like broadening of the wave packet due to forces exerted by an external potential and (ii) an intrinsically quantum-mechanical spreading dictated by the uncertainty principle. Due to the interference nature of quantum dynamics, the overall spreading is not a simple sum of the two contributions, but rather a more intricate process.

A natural question arises: Is there an intuitive and, at the same time, quantitative theoretical description of the phenomenon of quantum spreading? In this paper, we develop such a description, based on the short-wavelength approximation to quantum dynamics, for the simple system of a two-dimensional quantum wave packet scattered by a weak Gaussian potential. In particular, we show that the quantum scattering process bears a close mathematical analogy with the phenomenon of focusing (or defocusing) of light rays by a thin lens and can be described using the language of geometrical optics. Interestingly, on the quantum side, the use of the eikonal approximation [6], i.e., including interference of straight paths, yields the same thin lens equation as derived in classical optics from refracted light rays. Our theoretical approach provides an intuitive picture of the wave packet spreading [7,8] and its quantitative predictions are found to be in good agreement with the results of an exact numerical solution of the time-dependent Schrödinger equation.

\section{THEORY}

We consider a quantum particle of mass $m$ that evolves in the two-dimensional position space under the influence of the external potential

$$
V(\mathbf{q})=V_{0} e^{-\mathbf{q} \cdot \mathbf{A q}} .
$$

Here $V_{0}$ quantifies the strength of the potential, $\mathbf{q}$ is a column vector representing the particle's position, and $\mathbf{A}$ is a $2 \times 2$ orthogonal matrix with eigenvalues $a_{1}$ and $a_{2}$ corresponding, respectively, to orthonormal (column) eigenvectors $\mathbf{e}_{1}$ and $\mathbf{e}_{2}$. In other words,

$$
\mathbf{A}=\left(\mathbf{e}_{1} \mathbf{e}_{2}\right) \operatorname{diag}\left(a_{1}, a_{2}\right)\left(\mathbf{e}_{1} \mathbf{e}_{2}\right)^{T},
$$

where $\left|\mathbf{e}_{1}\right|=\left|\mathbf{e}_{2}\right|=1$ and $\mathbf{e}_{1} \cdot \mathbf{e}_{2}=0$. Hereinafter, the dot stands for the scalar product and the superscript $T$ denotes the matrix transposition. Equation (1) describes a Gaussian potential island centered at $\mathbf{q}=\mathbf{0}$ (see Fig. 1). The spatial extent of the island is characterized by the length $l_{1}=1 / \sqrt{a_{1}}$ in the direction of the vector $\mathbf{e}_{1}$ and by $l_{2}=1 / \sqrt{a_{2}}$ in the direction of $\mathbf{e}_{2}$.

The dynamics of the quantum particle is fully described by its time-dependent wave function $\Psi_{t}(\mathbf{q})$. The latter is related to the initial wave function $\Psi_{0}(\mathbf{q})$ by means of a propagator $K$ in accordance with

$$
\Psi_{t}(\mathbf{q})=\int d \mathbf{q}^{\prime} K\left(\mathbf{q}, \mathbf{q}^{\prime} ; t\right) \Psi_{0}\left(\mathbf{q}^{\prime}\right) .
$$

Here the $\mathbf{q}^{\prime}$ integration runs over the whole two-dimensional plane. In general, the propagator $K$ is determined by solving the time-dependent Schrödinger equation

$$
\left(i \hbar \frac{\partial}{\partial t}+\frac{\hbar^{2}}{2 m} \nabla_{\mathbf{q}}^{2}-V(\mathbf{q})\right) K\left(\mathbf{q}, \mathbf{q}^{\prime} ; t\right)=0,
$$




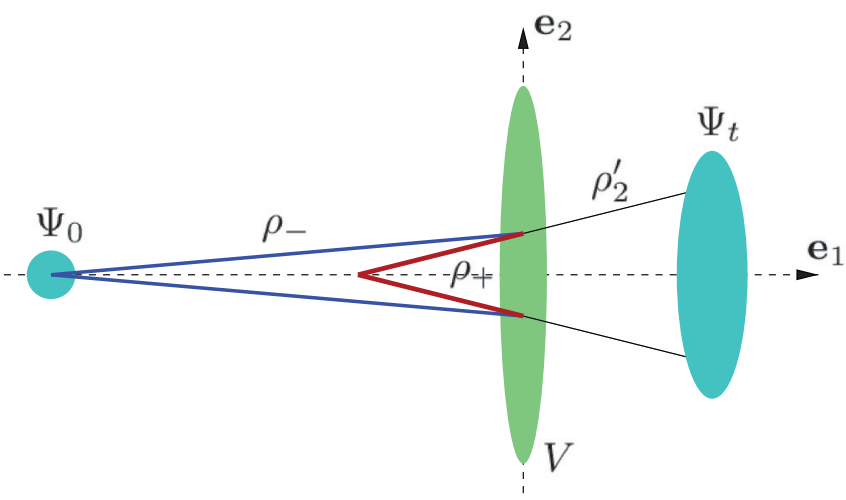

FIG. 1. (Color online) Schematic illustration of the scattering system under consideration.

subject to appropriate boundary and initial conditions (see, e.g., Ref. [9]). Generally, this task is analytically formidable and one is left to resort to numerical computations. In certain cases, however, analytical progress is made possible by constructing sensible approximations to the propagator. In this paper, we focus on one such approximation commonly referred to as semiclassics.

The semiclassical (or short-wavelength) approximation of the propagator $K\left(\mathbf{q}, \mathbf{q}^{\prime} ; t\right)$ is formulated in terms of classical trajectories $\gamma$ that start at the point $\mathbf{q}^{\prime}$ at time 0 and end at $\mathbf{q}$ at time $t$. More precisely, $\gamma=\{\mathbf{r}(\tau), \tau \in[0, t]\}$ such that $m \frac{d^{2} \mathbf{r}}{d \tau^{2}}+\nabla V(\mathbf{r})=\mathbf{0}$ with $\mathbf{r}(0)=\mathbf{q}^{\prime}$ and $\mathbf{r}(t)=\mathbf{q}$. The approximate propagator, commonly referred to as the Van Vleck-Gutzwiller propagator, can be written as [2]

$$
K\left(\mathbf{q}, \mathbf{q}^{\prime}, t\right)=\frac{\sqrt{D_{\gamma}}}{2 \pi i \hbar} \exp \left(\frac{i}{\hbar} S_{\gamma}-\frac{i \pi}{2} v_{\gamma}\right),
$$

where

$$
S_{\gamma} \equiv S[\mathbf{r}](t)=\int_{0}^{t} d \tau\left\{\frac{m}{2}\left(\frac{d \mathbf{r}}{d \tau}(\tau)\right)^{2}-V(\mathbf{r}(\tau))\right\}
$$

is Hamilton's principle function along the trajectory $\gamma$,

$$
D_{\gamma}=\left|\operatorname{det}\left(-\nabla_{\mathbf{q}^{\prime}} \nabla_{\mathbf{q}} S_{\gamma}\right)\right|
$$

is the stability factor of $\gamma$, and $v_{\gamma}$ is the so-called Maslov index, counting the number of conjugate points along $\gamma$; as far as our problem is concerned, the Maslov index is identically zero $v_{\gamma}=0$.

The approximation (5) is known to be reliable when applied to wave functions $\Psi_{0}(\mathbf{q})$ representing a quantum particle of sufficiently high kinetic energy $E_{0}$. We additionally assume that the Gaussian potential, given by Eq. (1), is weak compared to the particle's kinetic energy $\left|V_{0}\right| \ll E_{0}$. The validity of this condition is at the heart of our shallow potential island approximation corresponding to the eikonal approximation in scattering theory [6]. In this case (see the Appendix), $S_{\gamma}$ can be well approximated by $S_{\gamma_{0}}$, where $\gamma_{0}$ is the straight free-particle trajectory leading from $\mathbf{q}^{\prime}$ to $\mathbf{q}$ in time $t$, i.e., $\gamma_{0}=\{\mathbf{q} \tau / t+$ $\left.\mathbf{q}^{\prime}(1-\tau / t), \tau \in[0, t]\right\}$. Thus we write

$$
S_{\gamma} \simeq S_{\gamma_{0}}=\frac{m}{2 t}\left|\mathbf{q}-\mathbf{q}^{\prime}\right|^{2}-\int_{0}^{t} d \tau V\left(\mathbf{q}^{\prime}+\frac{\tau}{t}\left(\mathbf{q}-\mathbf{q}^{\prime}\right)\right) .
$$

The integral on the right-hand side of Eq. (8) can be straightforwardly evaluated to equal

$$
\begin{aligned}
& \frac{\sqrt{\pi} V_{0} t}{2 \mathcal{A}} \exp \left(-\frac{(\mathbf{q} \cdot \mathbf{A q})\left(\mathbf{q}^{\prime} \cdot \mathbf{A} \mathbf{q}^{\prime}\right)-\left(\mathbf{q} \cdot \mathbf{A} \mathbf{q}^{\prime}\right)^{2}}{\mathcal{A}^{2}}\right) \\
& \times\left[\operatorname{erf}\left(\frac{\mathbf{q} \cdot \mathbf{A}\left(\mathbf{q}-\mathbf{q}^{\prime}\right)}{\mathcal{A}}\right)-\operatorname{erf}\left(\frac{\mathbf{q}^{\prime} \cdot \mathbf{A}\left(\mathbf{q}-\mathbf{q}^{\prime}\right)}{\mathcal{A}}\right)\right],
\end{aligned}
$$

with

$$
\mathcal{A} \equiv \sqrt{\left(\mathbf{q}-\mathbf{q}^{\prime}\right) \cdot \mathbf{A}\left(\mathbf{q}-\mathbf{q}^{\prime}\right)} .
$$

Expression (9), and therefore Eq. (8), can be further simplified by taking into account the identity

$$
(\mathbf{q} \cdot \mathbf{A q})\left(\mathbf{q}^{\prime} \cdot \mathbf{A} \mathbf{q}^{\prime}\right)-\left(\mathbf{q} \cdot \mathbf{A} \mathbf{q}^{\prime}\right)^{2}=\left|\mathbf{q} \times \mathbf{q}^{\prime}\right|^{2} \operatorname{det} \mathbf{A},
$$

where $\times$ denotes the vector product. This yields

$$
\begin{aligned}
S_{\gamma} \simeq & \frac{m}{2 t}\left|\mathbf{q}-\mathbf{q}^{\prime}\right|^{2}-\frac{\sqrt{\pi} V_{0} t}{2 \mathcal{A}} \exp \left(-\frac{\left|\mathbf{q} \times \mathbf{q}^{\prime}\right|^{2} \operatorname{det} \mathbf{A}}{\mathcal{A}^{2}}\right) \\
& \times\left[\operatorname{erf}\left(\frac{\mathbf{q} \cdot \mathbf{A}\left(\mathbf{q}-\mathbf{q}^{\prime}\right)}{\mathcal{A}}\right)-\operatorname{erf}\left(\frac{\mathbf{q}^{\prime} \cdot \mathbf{A}\left(\mathbf{q}-\mathbf{q}^{\prime}\right)}{\mathcal{A}}\right)\right] .
\end{aligned}
$$

A substitution of Eq. (12) into Eq. (5) leads to an explicit closed-form expression for the semiclassical propagator $K\left(\mathbf{q}, \mathbf{q}^{\prime}, t\right)$ and therefore provides the complete solution of the time-dependent scattering problem in the short-wavelength regime.

The expression for the propagator $K\left(\mathbf{q}, \mathbf{q}^{\prime}, t\right)$ becomes especially simple and allows for an intuitive interpretation in the following special case. Let us consider a setup in which the receiver $\mathbf{q}$ and the source $\mathbf{q}^{\prime}$ lie on opposite sides of and at almost the same distance, large compared to $l_{1}$, from the center of the Gaussian scattering potential and in which the vector $\mathbf{q}-\mathbf{q}^{\prime}$ is nearly aligned with one of the principal directions (taken, for concreteness, to be $\mathbf{e}_{1}$ ) of the potential island. In other words, we are interested in the asymptotic form of the function $K\left(\mathbf{q}, \mathbf{q}^{\prime}, t\right)$ in the case that

$$
\mathbf{q}=L \mathbf{e}_{1}+\xi, \quad \mathbf{q}^{\prime}=-L \mathbf{e}_{1}+\xi^{\prime}
$$

and

$$
|\xi|,\left|\xi^{\prime}\right|, l_{1} \ll L .
$$

Substituting Eq. (13) into Eqs. (10) and (12), taking into account that $\operatorname{erf}( \pm z) \rightarrow \pm 1$ as $z \rightarrow+\infty$, and keeping only terms to the leading order in $|\xi| / L$ and $\left|\xi^{\prime}\right| / L$ in the argument of the exponential function, we obtain

$$
\begin{aligned}
S_{\gamma} \simeq & \frac{m}{2 t}\left|2 L \mathbf{e}_{1}+\boldsymbol{\xi}-\boldsymbol{\xi}^{\prime}\right|^{2} \\
& -\frac{\sqrt{\pi}}{2} \frac{l_{1}}{L} V_{0} t \exp \left(-\frac{\left|\mathbf{e}_{1} \times\left(\boldsymbol{\xi}+\boldsymbol{\xi}^{\prime}\right)\right|^{2}}{\left(2 l_{2}\right)^{2}}\right) .
\end{aligned}
$$

Then, using the basis representations $\xi=\xi_{1} \mathbf{e}_{1}+\xi_{2} \mathbf{e}_{2}$ and $\xi^{\prime}=$ $\xi_{1}^{\prime} \mathbf{e}_{1}+\xi_{2}^{\prime} \mathbf{e}_{2}$ in Eq. (15) and further assuming that

$$
\left|\xi_{2}\right|,\left|\xi_{2}^{\prime}\right| \ll l_{2}
$$

we write, approximately,

$$
S_{\gamma} \simeq-\frac{\sqrt{\pi}}{2} \frac{l_{1}}{L} V_{0} t+S_{\gamma}^{(1)}+S_{\gamma}^{(2)},
$$


where

$$
S_{\gamma}^{(1)}=\frac{m}{2 t}\left(2 L+\xi_{1}-\xi_{1}^{\prime}\right)^{2}
$$

and

$$
S_{\gamma}^{(2)}=\frac{m}{2 t}\left(\xi_{2}-\xi_{2}^{\prime}\right)^{2}+\frac{\sqrt{\pi}}{8} \frac{l_{1}}{L\left(l_{2}\right)^{2}} V_{0} t\left(\xi_{2}+\xi_{2}^{\prime}\right)^{2} .
$$

In view of Eqs. (17)-(19), the stability factor (7) along the trajectory $\gamma$ can be written as $D_{\gamma} \simeq D_{\gamma}^{(1)} D_{\gamma}^{(2)}$, with $D_{\gamma}^{(1)}=\left|-\partial_{\xi_{1}} \partial_{\xi_{1}^{\prime}} S_{\gamma}^{(1)}\right|=m / t$ and $D_{\gamma}^{(2)}=\left|-\partial_{\xi_{2}} \partial_{\xi_{2}^{\prime}} S_{\gamma}^{(2)}\right|=$ $m / t-\sqrt{\pi} l_{1} V_{0} t /\left[4 L\left(l_{2}\right)^{2}\right]$, provided

$$
t^{2} \ll \frac{m\left(l_{2}\right)^{2}}{\left|V_{0}\right|} \frac{L}{l_{1}}
$$

In fact, Eq. (20) states a necessary condition for Eq. (19) to constitute a healthy perturbative expansion of $S_{\gamma}^{(2)}$ in powers of $V_{0}$.

The full semiclassical propagator (5) can now be written as

$$
\begin{aligned}
K\left(\mathbf{q}, \mathbf{q}^{\prime}, t\right) \simeq & \exp \left(-i \frac{\sqrt{\pi}}{2} \frac{l_{1}}{L} \frac{V_{0} t}{\hbar}\right) \\
& \times K_{0}\left(L+\xi_{1},-L+\xi_{1}^{\prime} ; t\right) K_{V}\left(\xi_{2}, \xi_{2}^{\prime} ; t\right),
\end{aligned}
$$

where

$$
K_{0}\left(z, z^{\prime}, \tau\right) \equiv \sqrt{\frac{m}{2 \pi i \hbar \tau}} \exp \left(i \frac{m}{2 \hbar \tau}\left(z-z^{\prime}\right)^{2}\right)
$$

is the free-particle propagator describing the motion of the particle in the $\mathbf{e}_{1}$ direction, while

$$
\begin{aligned}
K_{V}\left(z, z^{\prime}, \tau\right) \equiv & K_{0}\left(z, z^{\prime}, \tau\right) \sqrt{1-\frac{\sqrt{\pi}}{4} \frac{l_{1}}{L} \frac{V_{0} \tau^{2}}{m\left(l_{2}\right)^{2}}} \\
& \times \exp \left(i \frac{\sqrt{\pi}}{8} \frac{l_{1}}{L} \frac{V_{0} \tau}{\hbar} \frac{\left(z+z^{\prime}\right)^{2}}{\left(l_{2}\right)^{2}}\right)
\end{aligned}
$$

accounts for the wave function spreading in the orthogonal $\mathbf{e}_{2}$ direction. Clearly, $K_{V} \rightarrow K_{0}$ as $V_{0} \rightarrow 0$, recovering the free-particle limit.

We now observe that

$$
\begin{aligned}
& K_{V}\left(z, z^{\prime}, \tau\right) \\
& =\int_{-\infty}^{+\infty} d \zeta K_{0}(z, \zeta, \tau / 2) \\
& \quad \times \exp \left[i \frac{\sqrt{\pi}}{2} \frac{l_{1}}{L} \frac{V_{0} \tau}{\hbar}\left(1-\frac{\sqrt{\pi}}{4} \frac{l_{1}}{L} \frac{V_{0} \tau^{2}}{m\left(l_{2}\right)^{2}}\right)^{-1} \frac{\zeta^{2}}{\left(l_{2}\right)^{2}}\right] \\
& \quad \times K_{0}\left(\zeta, z^{\prime}, \tau / 2\right) .
\end{aligned}
$$

Equation (24) is an identity and can be verified straightforwardly by evaluating the Gaussian integral on the right-hand side. Then, after Eq. (20) is taken into account, Eq. (24) reduces to

$$
\begin{aligned}
K_{V}\left(z, z^{\prime}, \tau\right) \simeq & \int_{-\infty}^{+\infty} d \zeta K_{0}(z, \zeta, \tau / 2) \\
& \times \exp \left[i \frac{\sqrt{\pi}}{2} \frac{l_{1}}{L} \frac{V_{0} \tau}{\hbar} \frac{\zeta^{2}}{\left(l_{2}\right)^{2}}\right] K_{0}\left(\zeta, z^{\prime}, \tau / 2\right)
\end{aligned}
$$

The physical picture offered by Eq. (25) is as follows. The propagator $K_{V}$, evolving a quantum state during time $\tau$, can be view as a result of three consecutive operations: (i) a free-particle propagation during time $\tau / 2$, (ii) an instantaneous phase change, or kick, of the quantum state, and (iii) another free-particle propagation during $\tau / 2$. This interpretation, as well as the physical meaning of the kick operator, becomes apparent when the evolving quantum state is given by a Gaussian wave packet. To this end, we consider as the initial state the two-dimensional wave packet

$$
\Psi_{0}\left(\xi_{1}^{\prime}, \xi_{2}^{\prime}\right) \equiv \psi_{0}^{(1)}\left(\xi_{1}^{\prime} ; \rho_{1}\right) \psi_{0}^{(2)}\left(\xi_{2}^{\prime} ; \rho_{2}\right)
$$

with

$$
\begin{gathered}
\psi_{0}^{(1)}(z ; \rho) \equiv\left(\frac{1}{\pi \sigma^{2}}\right)^{1 / 4} \exp \left[i \frac{m v}{\hbar}\left(\frac{z^{2}}{2 \rho}+z\right)\right], \\
\psi_{0}^{(2)}(z ; \rho) \equiv\left(\frac{1}{\pi \sigma^{2}}\right)^{1 / 4} \exp \left(i \frac{m v}{\hbar} \frac{z^{2}}{2 \rho}\right) .
\end{gathered}
$$

Here $\rho_{1}$ and $\rho_{2}$ (usually termed radii of curvature [10]) are two generally complex-valued parameters. The real-valued function $\sigma=\sigma(\rho)$, defined in accordance with

$$
\frac{1}{\sigma^{2}} \equiv \frac{m v}{\hbar} \operatorname{Im}\left(\frac{1}{\rho}\right)=-\frac{m v}{\hbar} \frac{\operatorname{Im} \rho}{|\rho|^{2}},
$$

where Im denotes the imaginary part, quantifies the positionspace dispersion of the wave packet. Furthermore, $v$ specifies the average velocity (and $m v$ the average momentum) of the particle.

Now, acting with the propagator (21) on the initial state (26), which is assumed to be spatially localized around the position vector $\mathbf{q}^{\prime}=-L \mathbf{e}_{1}$ (or around the origin in the $\boldsymbol{\xi}^{\prime}$-coordinate frame), we obtain the quantum state after time $t$ locally, in the vicinity of the point $\mathbf{q}=L \mathbf{e}_{1}$ (or around the origin in the $\xi$-coordinate frame):

$$
\Psi_{t}\left(\xi_{1}, \xi_{2}\right) \simeq \exp \left(-i \frac{\sqrt{\pi}}{2} \frac{l_{1}}{L} \frac{V_{0} t}{\hbar}\right) \psi_{t}^{(1)}\left(\xi_{1} ; \rho_{1}\right) \psi_{t}^{(2)}\left(\xi_{2} ; \rho_{2}\right),
$$

where

$$
\begin{gathered}
\psi_{t}^{(1)}(z ; \rho) \equiv \int_{-\infty}^{+\infty} d \zeta K_{0}(L+z,-L+\zeta, t) \psi_{0}^{(1)}(\zeta ; \rho) \\
\psi_{t}^{(2)}(z ; \rho) \equiv \int_{-\infty}^{+\infty} d \zeta K_{V}(z, \zeta, t) \psi_{0}^{(2)}(\zeta ; \rho)
\end{gathered}
$$

As we are concerned with the semiclassical limit, it is reasonable to expect that, in the course of its time evolution, the quantum wave packet remains concentrated around the corresponding classical trajectory. This means that the center of the wave packet, starting from the point $-L \mathbf{e}_{1}$ at time 0 , reaches the point $L \mathbf{e}_{1}$ in time $t$, such that

$$
v t=2 L \text {. }
$$

It is at this instant that $\Psi_{t}\left(\xi_{1}, \xi_{2}\right)$ is localized around the origin in the $\xi$-coordinate frame and that the propagator approximation, given by Eq. (21), proves the most useful.

Fixing the time $t$ in accordance with Eq. (33) and evaluating the Gaussian integral in Eq. (31), we obtain

$$
\psi_{t}^{(1)}\left(z ; \rho_{1}\right)=e^{i \phi_{1}} \psi_{0}^{(1)}\left(z ; \rho_{1}+v t\right),
$$


where $\phi_{1}=E_{0} t / \hbar-(1 / 2) \arg \left(1+v t / \rho_{1}\right)$, with

$$
E_{0} \equiv \frac{m v^{2}}{2}
$$

denoting the kinetic energy of the corresponding classical particle. The physical interpretation of Eq. (34) is that the $\mathbf{e}_{1}$ component of the wave packet retains its Gaussian shape as its center travels in space on top of the corresponding classical trajectory (in agreement with the Ehrenfest theorem). The spreading of the $\mathbf{e}_{1}$ component of the wave packet is entirely described by the linear transformation of the corresponding radius of curvature $\rho_{1} \rightarrow \rho_{1}+v t$ and, in the weak potential limit, this spreading is not affected by the external potential.

We now focus on the time evolution of the $\mathbf{e}_{2}$ component of the wave packet. As before, we keep the time $t$ fixed in accordance with Eq. (33). Substituting Eq. (25) into Eq. (32) and successively evaluating two Gaussian integrals, we obtain

$$
\psi_{t}^{(2)}\left(z ; \rho_{2}\right)=e^{i \phi_{2}} \psi_{0}^{(2)}\left(z ; \rho_{2}^{\prime}\right),
$$

where $\rho_{2}^{\prime}=\rho_{+}+v t / 2$,

$$
\frac{1}{\rho_{+}}=\frac{1}{\rho_{-}}+\frac{1}{f}
$$

$\rho_{-}=\rho_{2}+v t / 2$,

$$
f \equiv \frac{1}{\sqrt{\pi}} \frac{E_{0}}{V_{0}} \frac{\left(l_{2}\right)^{2}}{l_{1}},
$$

and $\phi_{2}=-(1 / 2)\left[\arg \left(\rho_{2}^{\prime} / \rho_{+}\right)+\arg \left(\rho_{-} / \rho_{2}\right)\right]$.

The physical picture of the wave-packet spreading, offered by the central equations (36)-(38), bears close analogy with the focusing (or defocusing) of light rays by a thin optical lens. Indeed, the well-known thin lens equation (object distance) $)^{-1}+$ (image distance $)^{-1}=(\text { focal length })^{-1}$ can be readily recovered from Eq. (37) by interpreting $-\rho_{-}$and $\rho_{+}$as the distances from, respectively, the object and its image to a lens of the focal length $f$; the role of the lens is played here by the Gaussian potential island (see Fig. 1).

It is important to point out that the above analogy between the wave packet scattering in quantum mechanics and the ray focusing in optics is not a trivial one: In the quantummechanical case, the distances $-\rho_{-}$and $\rho_{+}$are intrinsically complex valued and can only be related to the true distances, encountered in optics, in a nonlinear way.

Finally, we note that our simple wave-packet-propagation construction can be generalized to arbitrary times $t$. This generalization can be summarized as follows. Suppose that the initial wave packet is centered around a point with coordinates $(Q, 0)$, where $Q<0$, and that the initial wave function is given by the product $\psi_{0}^{(1)}\left(q_{1}-Q ; \rho_{1}\right) \psi_{0}^{(2)}\left(q_{2} ; \rho_{2}\right)$. Then, at a later time $t$, the wave function is given, up to an overall phase factor, by $\psi_{0}^{(1)}\left(q_{1}-Q-v t ; \rho_{1}+v t\right) \psi_{0}^{(2)}\left(q_{2} ; \rho_{2}^{\prime}\right)$, where

$$
\rho_{2}^{\prime}= \begin{cases}\rho_{2}+v t, & t<|Q| / v \\ \rho_{+}+v(t-|Q| / v), & t \geqslant|Q| / v\end{cases}
$$

and $\rho_{+}$is determined from Eq. (37) with $\rho_{-}=\rho_{2}+|Q|$.

\section{NUMERICAL CONFIRMATION}

We now confirm the validity of the quantum lens formulas, given by Eqs. (37)-(39), by comparing their predictions to results of numerical simulations. The latter were performed by solving the time-dependent Schrödinger equation, governing the evolution of the wave packet, numerically, using the method of expanding the propagator in a series of Chebyshev polynomials of the Hamiltonian. The reader is referred to Refs. [11-13] for a comprehensive description of the method and its implementations.

The concrete system that we consider is schematically illustrated in Fig. 1 and described by the following set of parameters. Hereinafter, we adopt atomic units $\hbar=m=1$. The external Gaussian potential (1) is characterized by $l_{1}=0.1$ and $l_{2}=1$ and the potential strength $V_{0}$ plays the role of a variable parameter, with values ranging between 10 and 40. The initial state of the particle is given by the wave function $\psi_{0}^{(1)}\left(q_{1}-Q ; \rho_{1}\right) \psi_{0}^{(2)}\left(q_{2} ; \rho_{2}\right)$, with $Q=-0.8, v=$ 60 , and $\rho_{1}=\rho_{2}=-i(m v / \hbar)\left(\sigma_{0}\right)^{2}$, where $\sigma_{0}=0.1$ quantifies the initial position-space dispersion of the wave packet [see Eq. (29)]. Note that the kinetic energy of the classical particle $E_{0}=m v^{2} / 2=1800$ is large compared to the strength of the external potential.

Our aim is to study the wave-packet spreading in the direction orthogonal to the propagation direction. This spreading is given by the time dependence of the dispersion along the $\mathbf{e}_{2}$ axis,

$$
\sigma_{2}=\sqrt{2 \int d \mathbf{q}\left(q_{2}\right)^{2}\left|\Psi_{t}(\mathbf{q})\right|^{2}} .
$$

[Note that, due to the symmetry of $\Psi_{t}(\mathbf{q})$ under the reflection $q_{2} \rightarrow-q_{2}$, the expectation value of $q_{2}$ is zero, i.e., $\int d \mathbf{q} q_{2}\left|\Psi_{t}(\mathbf{q})\right|^{2}=0$.] For our choice of the initial state, $\sigma_{2}=\sigma_{0}$ at $t=0$ and $\sigma_{2}$ increases as a function of time. In the limit $V_{0}=0$, this increase is determined by the free-particle

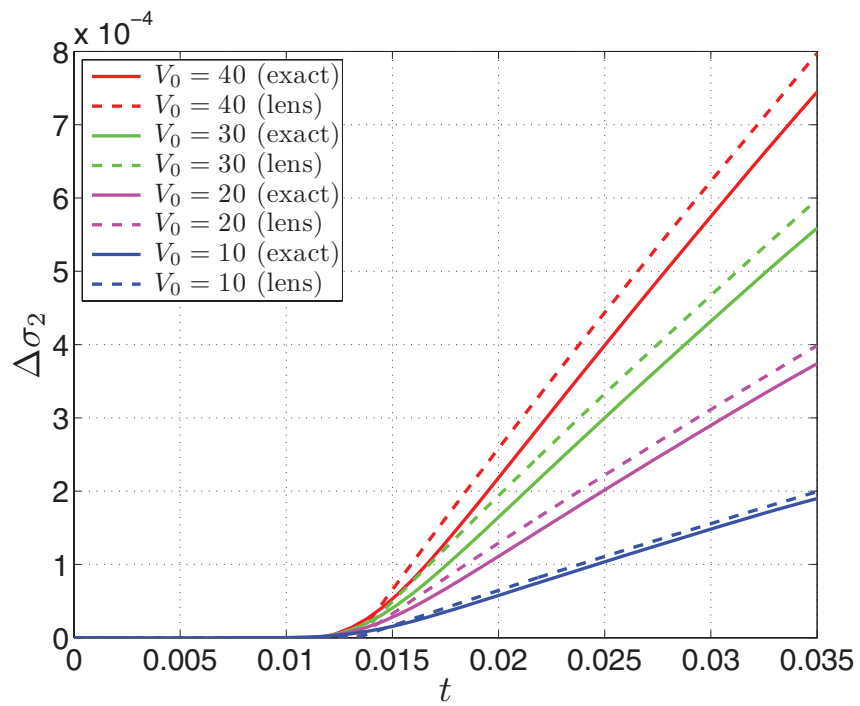

FIG. 2. (Color online) Spreading of the wave packet in the direction orthogonal to the direction of propagation. See the text for details. 
spreading $\sigma_{2}^{\text {free }}=\sigma\left(\rho_{2}+v t\right)$, with the function $\sigma(\rho)$ defined by Eq. (29). A straightforward calculation yields

$$
\sigma_{2}^{\text {free }}=\sqrt{\left(\sigma_{0}\right)^{2}+\left(\frac{\hbar t}{m \sigma_{0}}\right)^{2}} .
$$

In the case $V_{0} \neq 0$, the external potential causes additional spreading that can be quantified by

$$
\Delta \sigma_{2} \equiv \sigma_{2}-\sigma_{2}^{\text {free }} .
$$

Figure 2 shows the dependence of $\Delta \sigma_{2}$ on time $t$ for four different potential strengths $V_{0}=10$ (blue), 20 (magenta), 30 (green), and 40 (red). The solid curves represent the results of the exact numerical solution of the time-dependent Schrödinger equation, while the dashed curves show the analytical lens approximation, namely, $\sigma\left(\rho_{2}^{\prime}\right)-\sigma_{2}^{\text {free }}$ with $\rho_{2}^{\prime}$, calculated in accordance with Eqs. (37)-(39). As expected, $\Delta \sigma_{2} \simeq 0$ for $t \lesssim|Q| / v \simeq 0.013$, corresponding to the time that it takes for the classical particle to reach the potential island. Figure 2 shows the theoretical predictions to be in reasonable agreement with the numerical results. It also confirms that the agreement improves as the potential strength is decreased.

\section{CONCLUSION}

In this paper, we have constructed an approximate analytical solution to the problem of scattering of a localized quantum wave packet by a weak Gaussian potential. Our solution is valid in the semiclassical regime, in which the particle's de Broglie wavelength can be considered short compared to all other length scales of the system. We have shown that the quantum scattering process is closely analogous to the phenomenon of focusing (or defocusing) of light rays by a thin optical lens. In particular, the mathematical formula quantifying the wave-packet spreading [Eq. (37)] is largely equivalent to the thin lens formula of geometrical optics. The main difference between the thin lens formula in optics and Eq. (37) is that the former operates with true real-valued distances from the lens to the object and to the image, while the distances $\rho_{-}$ and $\rho_{+}$entering Eq. (37) are intrinsically complex valued. It is only in the classical limit $m v / \hbar \rightarrow \infty$ that $\rho_{-}$and $\rho_{+}$become real valued and the optical thin lens formula is recovered.

It is instructive to further compare the ray optics picture with our quantum-mechanical result. The thin lens formula in optics, which is the classical limit of Eq. (37), effectively describes the deflection, or bending, of light rays (classical trajectories), induced by the lens (external potential). However, only straight trajectories have been used in our semiclassical derivation of Eq. (37). This seeming paradox is resolved by the following argument, originally presented in Ref. [14] and for the readers' convenience reproduced in the Appendix. The main building block of the semiclassical propagator (5) is Hamilton's principal function along the classical trajectory connecting the initial and final points of the propagation. However, in a sufficiently weak external potential, the value of Hamilton's principal function along the true (generally bent) classical trajectory is very close to that along the corresponding straight trajectory, making the precise geometrical shape of the trajectory unsubstantial.

The approach taken in this paper is conceptually similar to the one used in Ref. [10] to analyze the spreading of quantum wave packets in the Lorentz gas. The latter consists of a particle moving in an array of fixed elastic scatterers, taken to be hard disks (spheres) in two (three) spatial dimensions. However, it is important to point out that in the Lorentz gas, unlike in the system addressed in the present paper, one must take into account deflections of classical trajectories in order to obtain the quantum-mechanical equivalent of the circular (spherical) mirror formula.

\section{ACKNOWLEDGMENTS}

The authors thank Tobias Kramer for useful discussions. A.G. acknowledges the hospitality of the University of Regensburg during a two-month visit where much of this work was done. K.R. thanks the Deutsche Forschungsgemeinschaft for financial support within Research Unit FOR 760.

\section{APPENDIX: EXPANSION OF HAMILTON'S PRINCIPAL FUNCTION}

The following discussion is based on the argument that was presented in Ref. [14].

Let us consider the Lagrangian

$$
\mathcal{L}_{\epsilon}[\mathbf{r}](\tau)=\frac{m}{2}\left(\frac{d \mathbf{r}}{d \tau}(\tau)\right)^{2}-\epsilon V(\mathbf{r}(\tau))
$$

along a trajectory $\mathbf{r}(\tau)$. Here $\epsilon \ll 1$ serves as a dimensionless strength of the potential. The corresponding Hamilton principal function is given by

$$
S_{\epsilon}[\mathbf{r}](t)=\int_{0}^{t} d \tau \mathcal{L}_{\epsilon}[\mathbf{r}](\tau) .
$$

We now denote by $\mathbf{r}_{\epsilon}(\tau)$ a trajectory that satisfies the boundary conditions $\mathbf{r}_{\epsilon}(0)=\mathbf{q}^{\prime}$ and $\mathbf{r}_{\epsilon}(t)=\mathbf{q}$ and makes the action $S_{\epsilon}[\mathbf{r}](t)$ stationary, i.e.,

$$
\frac{\delta S_{\epsilon}}{\delta \mathbf{r}}\left[\mathbf{r}_{\epsilon}\right](\tau)=\mathbf{0} .
$$

Then, for a trajectory $\mathbf{r}_{0}(\tau)$ that satisfies the same boundary conditions $\mathbf{r}_{0}(0)=\mathbf{q}^{\prime}$ and $\mathbf{r}_{0}(t)=\mathbf{q}$ and is the stationary trajectory of $S_{0}[\mathbf{r}](t)$, we have

$$
\begin{aligned}
S_{\epsilon}\left[\mathbf{r}_{0}\right](t)= & S_{\epsilon}\left[\mathbf{r}_{\epsilon}\right](t)+\int_{0}^{t} d \tau \frac{\delta S_{\epsilon}}{\delta \mathbf{r}}\left[\mathbf{r}_{\epsilon}\right] \cdot\left(\mathbf{r}_{0}-\mathbf{r}_{\epsilon}\right) \\
& +\frac{1}{2} \int_{0}^{t} d \tau\left(\mathbf{r}_{0}-\mathbf{r}_{\epsilon}\right) \cdot \frac{\delta^{2} S_{\epsilon}}{\delta \mathbf{r}^{2}}\left[\mathbf{r}_{\epsilon}\right]\left(\mathbf{r}_{0}-\mathbf{r}_{\epsilon}\right)+\cdots
\end{aligned}
$$

Substituting Eq. (A3) into (A4) and taking into account $\mathbf{r}_{\epsilon}=$ $\mathbf{r}_{0}+O(\epsilon)$, we obtain

$$
S_{\epsilon}\left[\mathbf{r}_{\epsilon}\right](t)=S_{\epsilon}\left[\mathbf{r}_{0}\right](t)+O\left(\epsilon^{2}\right) .
$$


[1] L. de Broglie, The Wave Nature of the Electron, Nobel Lecture (1929), available online http://nobelprize.org.

[2] M. C. Gutzwiller, Chaos in Classical and Quantum Mechanics (Springer, New York, 1990).

[3] H.-J. Stöckmann, Quantum Chaos: An Introduction (Cambridge University Press, Cambridge, 1999).

[4] F. Haake, Quantum Signatures of Chaos (Springer, Berlin, 2010).

[5] See, e.g., D. J. Tannor, Introduction to Quantum Mechanics: A Time-Dependent Perspective (Palgrave Macmillan, New York, 2007).

[6] J. J. Sakurai, Modern Quantum Mechanics (Addison-Wesley, Reading, MA, 1994), pp. 392-394.

[7] For related work see G. Vandegrift, Am. J. Phys. 72, 404 (2004).
[8] For an account of various semiclassical Gaussian wave-packet approaches see, e.g., E. J. Heller, Acc. Chem. Res. 39, 127 (2006).

[9] G. Barton, Elements of Green's Functions and Propagation (Clarendon, Oxford, 1989).

[10] A. Goussev and J. R. Dorfman, Phys. Rev. E 71, 026225 (2005).

[11] H. Tal-Ezer and R. Kosloff, J. Chem. Phys. 81, 3967 (1984).

[12] H. De Raedt, J. S. Kole, K. F. L. Michielsen, and M. T. Figge, Comput. Phys. Commun. 156, 43 (2003).

[13] W. van Dijk, J. Brown, and K. Spyksma, Phys. Rev. E 84, 056703 (2011).

[14] O. Bohigas, M.-J. Giannoni, A. M. O. de Almeida, and C. Schmit, Nonlinearity 8, 203 (1995). 